\title{
Biological responses to asbestos inhalation and pathogenesis of asbestos-related benign and malignant disease
}

\author{
Eduardo Solbes, Richart W Harper
}

Internal Medicine - Division of Pulmonary and Critical Care Medicine, UC Davis Medical Center, Sacramento, California, USA

\section{Correspondence to} Dr Eduardo Solbes, Internal Medicine - Division of Pulmonary and Critical Care Medicine UC Davis Medical Center, Sacramento, California 95817, USA; esolbes@ucdavis.edu

Accepted 11 December 2017

Published Online First 6 January 2018

\begin{abstract}
Asbestos comprises a group of fibrous minerals that are naturally occurring in the environment. Because of its natural properties, asbestos gained popularity for commercial applications in the late 19th century and was used throughout the majority of the 20th century, with predominant use in the construction, automotive, and shipbuilding industries. Asbestos has been linked to a spectrum of pulmonary diseases, such as pleural fibrosis and plaques, asbestosis, benign asbestos pleural effusion, small cell lung carcinoma, non-small cell lung carcinoma, and malignant mesothelioma. There are several mechanisms through which asbestos can lead to both benign and malignant disease, and they include alterations at the chromosomal level, activation of oncogenes, loss of tumor suppressor genes, alterations in cellular signal transduction pathways, generation of reactive oxygen and nitrogen species, and direct mechanical damage to cells from asbestos fibers. While known risk factors exist for the development of asbestos-related malignancies, there are currently no effective means to determine which asbestos-exposed patients will develop malignancy and which will not. There are also no established screening strategies to detect asbestos-related malignancies in patients who have a history of asbestos exposure. In this article, we present a case that highlights the different biological responses in human hosts to asbestos exposure.
\end{abstract}

\section{CASE DESCRIPTION}

A 54-year-old woman with mild persistent asthma presented to pulmonary clinic with a chief complaint of chronic pleurisy. She had indirect exposure to asbestos from her father, who worked in a shipyard and had daily asbestos exposure. The patient's mother did not have occupational exposure to asbestos, but would do her husband's laundry routinely. As an infant, the patient would be next to her mother in a playpen while her mother did the laundry. The patient's father had no sequelae from asbestos exposure, but her mother died from mesothelioma. The patient's chest imaging showed densely calcified bilateral pleural plaques (figure 1). There was no pulmonary fibrosis or pleural effusion. Due to ongoing pleurisy, she was referred to cardiothoracic surgery and underwent robotic-assisted thoracoscopic pleural plaque excision with mechanical pleurodesis. Histopathology of the excised plaques showed dense fibrosis. Since that time, the patient continues to be monitored in pulmonary clinic for sequelae from asbestos exposure. Given the patient's previous asbestos exposure and family history, the biggest concern is that she will develop mesothelioma. In the context of this clinical question, we review the various cellular, chemical, and molecular responses to asbestos exposure, the spectrum of asbestos-related disease, and the risk factors for developing asbestos-related malignancy .

\section{BACKGROUND}

Asbestos comprises a group of fibrous minerals that occur naturally in low levels in the air, water, and soil. Asbestos is predominantly divided into two groups, serpentine and amphibole, based on the characteristics of the fibers (figure 2). Serpentine asbestos contains the mineral chrysotile and gets its name from the curly, interwoven structure of its fibers. Amphibole asbestos is a mixed group of fibers that may contain actinolite, tremolite, anthophyllite, crocidolite, or amosite. Amphibole asbestos fibers are straight, needle-like, and more brittle than serpentine fibers, which limits its commercial applicability compared with serpentine asbestos. ${ }^{12}$ All asbestos fibers are chemically inert, durable, resistant to heat and fire, and do not conduct electricity. Because of these natural properties, asbestos gained popularity for commercial applications in the late 1800s, with predominant use in the construction, automotive, and shipbuilding industries. Due to increased shipbuilding during World War II, asbestos use increased substantially during the early 1940s.

Given the ubiquitous presence of asbestos in the environment, everyone is potentially exposed to asbestos. However, this low level of environmental exposure has not been shown to be a significant contributor to human disease. In contrast, millions of Americans with occupational exposure to asbestos, which peaked from the 1940s through the 1970s, have suffered from non-malignant diseases, such as asbestosis and benign pleural disease, as well 

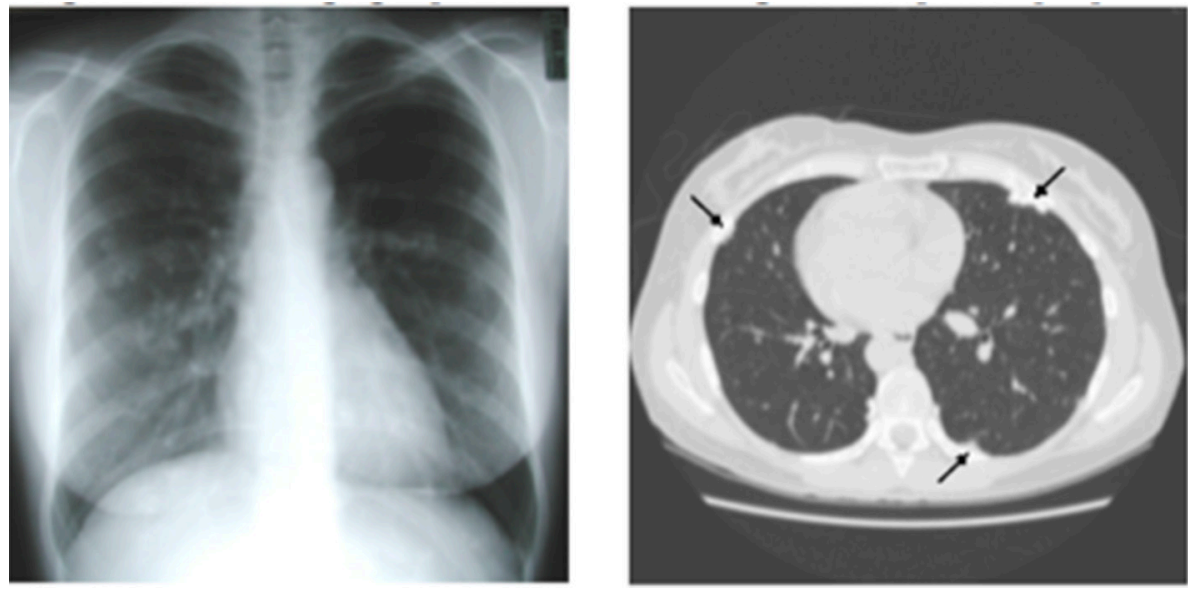

Figure 1 Chest imaging of patient demonstrating bilateral pleural plaques.

as malignancies associated with asbestos exposure, mainly mesothelioma and bronchogenic carcinoma. Because of these findings, the US Department of Health and Human Services, the Environmental Protection Agency (EPA), and the International Agency for Research on Cancer classify asbestos as a human carcinogen. The EPA banned all new uses of asbestos in 1989, and as a result, there has been a major decline in the use of asbestos in the USA. ${ }^{1}$

\section{SPECTRUM OF ASBESTOS-RELATED PLEUROPULMONARY DISEASE}

The host of cellular and molecular effects induced by asbestos fibers are thought to lead to the broad spectrum of pulmonary diseases that affect the lung parenchyma as well as the pleura. Asbestos has been determined to be a causative agent in both benign and malignant lung disease. This includes pulmonary and pleural fibrosis, pleural plaques, benign asbestos pleural effusion (BAPE), and malignancy (bronchogenic carcinoma and malignant mesothelioma) (box 1). An in-depth discussion regarding each of these diseases is beyond the scope of this article; however, each of these diseases will be briefly reviewed.
Asbestos-related pleural plaques and fibrosis are the most common radiographic findings among asbestos-exposed individuals, accounting for $>90$ per cent of asbestos-related pleural abnormalities. ${ }^{3}$ The development of pleural fibrosis depends on the cumulative dose of asbestos exposure and the elapsed time since the index exposure. ${ }^{4}$ Several studies have shown that pleural fibrosis is an independent contributor to restrictive lung disease as well as impairment in diffusing capacity of carbon monoxide, even in the absence of interstitial fibrosis. ${ }^{5-8}$ Diffuse pleural fibrosis and pleural plaques are thought to be distinct entities that have distinct underlying pathophysiology and their own unique radiographic characteristics. ${ }^{9}$ Pleural plaques are circumscribed acellular deposits of collagen that involve the parietal pleura. They typically are bilateral, symmetric, and usually involve the fifth to eighth ribs in the posterolateral portion of the thorax. The apices and costophrenic angles are usually spared. ${ }^{9}$ Diffuse pleural fibrosis, also referred to as pleural thickening, involves the visceral pleura. It may be the result of a previous pleural effusion and is commonly unilateral and involves the costophrenic angle. ${ }^{9}$

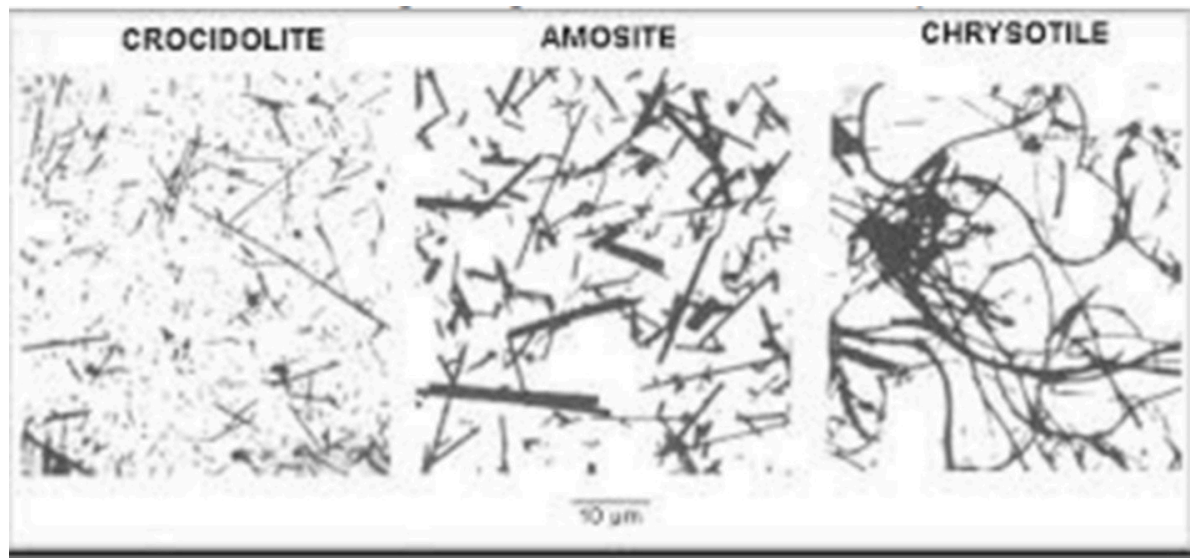

Figure 2 Electron micrograph highlighting the differences between the brittle, needle-like features of amphibole asbestos (crocidolite and amosite) versus the curly, interwoven serpentine asbestos (chrysotile) (downloaded from Google Images, original reference not available). 
Box 1 Spectrum of asbestos-related

pleuropulmonary disease

Pleural fibrosis and pleural plaques.

Asbestosis.

- Benign asbestos pleural effusion.

- Small cell and non-small cell lung carcinoma.

- Malignant mesothelioma.

The pathogenesis of both entities is not well understood. The formation of pleural plaques is suggested to occur via migration of asbestos microfibrils to the parietal pleura through the parenchymal lymphatic system or through the intercostal blood vessels. Once in the parietal pleura, an inflammatory reaction ensues, which is either caused by the asbestos microfibril itself or by agents that have been co-transported with the microfibril. ${ }^{10}$ Diffuse pleural thickening is thought to arise as interstitial parenchymal fibrosis extends peripherally to the visceral pleura. ${ }^{9}$ The migration of asbestos microfibrils to the periphery of the lung with subsequent development of inflammation and fibrosis of pleural lymphatics has been shown in both animal and human studies. ${ }^{11-13}$ Alternatively, fiber deposition may induce chronic, recurring hemorrhagic pleural effusions leading to a localized inflammatory reaction in the visceral pleura and subsequent pleural fibrosis. ${ }^{14} 15$

Asbestosis is a form of interstitial lung disease that is characterized by diffuse and bilateral interstitial fibrosis. ${ }^{9}$ As with pleural fibrosis, there is a dose-response relationship between exposure to asbestos and risk of developing asbestosis. The latency period between initial exposure and the onset of disease is typically $15-20$ years. ${ }^{9}$ The interstitial fibrosis is typically visible on chest X-ray, though in some cases the interstitial fibrosis is only visible by high-resolution CT scan (HRCT). Additional findings occasionally seen on HRCT in patients with asbestosis include cystic changes, parenchymal bands, honeycombing, and ground glass opacities (suggesting an ongoing inflammatory component to the disease). ${ }^{9}$ The diagnosis of asbestosis requires a history of asbestos exposure and the demonstration of interstitial fibrosis on chest imaging. In 90 per cent of cases, the diagnosis of asbestosis is made on these factors alone. ${ }^{9}$ The American Thoracic Society and American Medical Association currently recommend against lung biopsy if the sole reason to perform biopsy is to document the histopathological presence of asbestos fibers within the lung. ${ }^{16}{ }^{17}$ Lung biopsy remains an important diagnostic tool if the etiology of the pulmonary fibrosis is in question.

Asbestosis, pleural plaques, and pleural fibrosis are benign entities whose natural history is typically that of slowly progressive disease. Progression is associated with advanced age, more extensive occupational exposure to asbestos, tobacco smoking, and increased radiographic evidence of asbestos-related disease. ${ }^{18}$ Disease progression is typically manifested as worsening restrictive physiology and worsening impairment in diffusing capacity for carbon monoxide on pulmonary function tests. ${ }^{9}$ Clinical symptoms and signs of progressive disease include increasing dyspnea with exertion, declining exercise capacity, crackles on lung auscultation, fingernail clubbing, and development of hypoxemia necessitating the use of supplemental oxygen. At the present time, no specific pharmacotherapy exists for pleural plaques, pleural fibrosis, or asbestosis. No clinical trials have been performed to evaluate the role of specific therapies for these diseases. ${ }^{9}$ The current hallmarks of therapy include tobacco smoking cessation, early identification of disease to prevent any further occupational asbestos exposure, use of supplemental oxygen if hypoxemia is present, early treatment of respiratory infections, and vaccination against influenza and pneumococcal pneumonia.

BAPEs typically are unilateral and small. ${ }^{15}$ Symptoms of BAPE are non-specific and may include dyspnea, cough, pleuritic chest pain, and uncommonly, fever. They have also been reported to be asymptomatic in 66 per cent of cases. ${ }^{15}$ They typically precede the onset of interstitial disease by several years. ${ }^{15}$ There is not a clear dose-response correlation to asbestos exposure and BAPE. Pleural fluid analysis in BAPE will reveal an exudative effusion. The appearance can vary from a serous, serosanguinous, or frankly bloody fluid. ${ }^{19}$ Pleural fluid eosinophilia (with eosinophil counts up to 50 per cent of total nucleated cells) are sometimes be seen. ${ }^{19}$ BAPE is often seen in conjunction with other radiographic stigmata of chronic asbestos exposure. In one study, BAPE was more commonly associated with asbestosis, pleural plaques, rounded atelectasis, and diffuse pleural thickening compared with malignant mesothelioma. ${ }^{20}$

Malignant diseases associated with asbestos include small cell lung carcinoma, non-small cell lung carcinoma (collectively referred to as bronchogenic carcinoma), and mesothelioma. Malignant mesothelioma arises from mesothelial cells in the pleura, peritoneum, pericardium, and tunica vaginalis. Risk factors for developing mesothelioma will be discussed separately. Mesothelioma remains rare in the general population, with an estimated annual incidence of death of 1.75 per million in the USA between 1987 and $1996 .{ }^{4}$ The prognosis overall remains poor, with limited treatment options available. Further discussion about bronchogenic carcinoma is beyond the scope of this review.

\section{CELLULAR AND MOLECULAR EFFECTS OF ASBESTOS INHALATION}

Asbestos exposure can occur through inhalation, ingestion, or dermal contact, with inhalation being the most prevalent and clinically significant route of exposure. ${ }^{4}$ There are five mechanisms through which asbestos fibers deposit in the respiratory tract: impaction, sedimentation, interception, electrostatic precipitation, and diffusion. Impaction and sedimentation are dependent on the diameter of the fiber, interception is determined by the length of the fiber, and electrostatic precipitation is due to the electrical charge of the fiber. ${ }^{9}$ Amphibole fibers, which are uniform in distribution and arrangement, align parallel to the axis of airflow and typically deposit in alveolar ducts. Serpentine fibers, which are heterogeneous in arrangement, have a mixed flow pattern and will usually deposit in airway bifurcations. ${ }^{9}$ Fibers that land in ciliated portions of the airways are typically removed via the mucociliary escalator and do not result in lung injury. ${ }^{4}$ While all types of asbestos may have deleterious health effects, amphibole asbestos is thought to be more harmful to human health than serpentine asbestos. ${ }^{1}$ 
The precise mechanisms to explain fiber toxicity are poorly understood and may be a consequence of the differential deposition pattern, surface reactivity, or biodurability of these fibers. In addition, genetics likely play an important role in disease development.

The primary pathogenic pathways thought to induce asbestos-related fibrosis or malignant transformation include inflammation, apoptosis, and oxidative stress. Direct injury to epithelial cells lining the airways and alveoli, the first cells that come into contact with inhaled asbestos fibers, occurs shortly after contact with asbestos fibers. In rat models, asbestos fibers have been shown to deposit preferentially at alveolar duct bifurcations. ${ }^{21}$ These bifurcations are often the earliest sites of fibrosis. ${ }^{21} 22$ In humans, the pattern of lung epithelial injury has been shown to occur through both cytolytic and non-cytolytic mechanisms. Increased permeability of the epithelium can lead to fibrosis through increased movement of inflammatory mediators from the alveoli into the interstitium and by causing collapse of small airways and alveolar ducts. ${ }^{2324}$ Epithelial cells as well as alveolar macrophages phagocytize impacted asbestos fibers, ${ }^{21}{ }^{25}$ which is associated with increased permeability of the epithelium and epithelial injury. ${ }^{26}{ }^{27}$ After phagocytosis, fibers are transported along a microtubule network to the perinuclear region inducing further inflammation and damage. ${ }^{28}$

In addition to direct mechanical injury, asbestos can generate reactive oxygen species (ROS) that induce further damage. ${ }^{29}$ Generation of ROS occurs through various mechanisms. One mechanism is a direct effect from the asbestos fibers themselves. Iron on the asbestos fibers serves as a catalyst for the Fenton reaction, which generates a highly toxic hydroxyl radical from hydrogen peroxide. ${ }^{30}$ This is directly damaging to cells and will activate an inflammatory cascade inducing further damage. ${ }^{29}$ ROS can cause DNA strand breakage in mesothelial cells, which can increase the risk of developing malignancy. ${ }^{31}$ In addition to ROS, there is also evidence that reactive nitrogen species (RNS) play a role in asbestos-related lung injury. Inhaled asbestos fibers can induce the activity of inducible nitric oxide synthase in alveolar macrophages, A549 cells (a cell line derived from a human lung cancer cell line), and mesothelial cells. Nitric oxide can then interact with $\mathrm{O}_{2}$ to form peroxynitrate (NOOO), which is a potent oxidizing compound. ${ }^{32-34}$ The precise mechanisms whereby RNS cause lung injury are uncertain and are open for further investigation. ${ }^{9}$

Asbestos has been found to be active in signal transduction pathways in lung epithelial cells and mesothelial cells important for fibrosis or malignant transformation. ${ }^{35}$ Numerous cell membrane-mediated signal transduction pathways are regulated by activation of phospholipase $\mathrm{C}$ or $\mathrm{D}$. When these are activated, the result is increased intracellular calcium and increased protein kinase $\mathrm{C}$ (PKC) activity. PKC translocates to the cell membrane when activated and mediates numerous cellular functions, including regulation of gene expression, cellular growth, cellular shape, and epithelial permeability. ${ }^{378}$ Studies using hamster tracheal epithelial cells have shown that crocidolite asbestos exposure increases both phospholipase $\mathrm{C}$ and PKC activity. ${ }^{35}$ Asbestos has also been shown to activate tyrosine kinases, which promote cell proliferation, growth factor signal transduction, and alterations in cell-cell adherence.
Box 2 Cellular and molecular effects of asbestos inhalation

Direct mechanical injury.

- Generation of reactive oxygen and nitrogen species.

- Alteration of cellular signal transduction pathways.

- Loss of tumor suppressor genes and activation of oncogenes.

- Chromosomal and DNA alteration.

Silica, an important component of asbestos fibers, has also been shown to increase tyrosine kinase activity in human lung macrophages. ${ }^{39}$

Similarly, asbestos exposure is associated with activation of oncogenes and loss of tumor suppressor genes. Oncogenes play a central role in cell proliferation and cell transformation. Experiments with rat pleural mesothelial cells have shown that asbestos induces persistent elevation of $c$-fos and $c$-jun mRNA. ${ }^{40}$ Expression of $c$-fos and $c-j u n$ is required in the cell cycle for transition from the G1 phase (cell growth) into the $S$ phase (DNA replication). ${ }^{41}$ Increased expression of $c-j u n$ has been linked to cell transformation in hamster tracheal epithelial cells. ${ }^{40}$ Crocidolite asbestos exposure has been shown to result in persistent nuclear-factor- $\mathrm{\kappa B}$ (NF- $\mathrm{kB}$ ) expression (box 2). ${ }^{42} \mathrm{NF}-\mathrm{\kappa B}$ is a transcription factor that regulates numerous genes that are involved in both cell proliferation and inflammatory pathways. C-myc, an oncogene involved in the pathogenesis of many cancers, is upregulated by NF- $\mathrm{\kappa B} .{ }^{42}$

\section{RISK FACTORS FOR DEVELOPING MESOTHELIOMA}

The link between inhalation of asbestos fibers and mesothelioma is well documented in both occupational exposures in humans and experimental exposures in animal studies. However, not all patients exposed to significant amounts of asbestos will develop disease. There are numerous factors that will determine whether or not an asbestos-exposed individual will develop asbestos-related disease. Such factors include level and duration of exposure, age during exposure, time since exposure, type of asbestos (chrysotile vs amphibole), fiber length/diameter, route of exposure (inhalation, ingestion, dermal), the host immune reaction to asbestos, and genetics. ${ }^{2}$

Of particular importance to cancer pathogenesis is the diameter and length of the fiber. ${ }^{2}$ Strong evidence exists from animal inhalational studies, intrathoracic dosing studies, and in vitro studies that long fibers are more carcinogenic than short fibers. ${ }^{4}$ In the lung, short fibers typically have shorter clearance half times than longer fibers. For this reason, amphibole fibers are not cleared from the lung as easily as chrysotile fibers. All types of asbestos fibers have been associated with mesothelioma. However, several studies suggest that amphibole fibers are more potent than chrysotile fibers at inducing mesothelioma. Specifically, mesothelioma is highly associated with exposure to asbestos fiber length $>5 \mu \mathrm{m}$. $^{4}$

Direct and indirect DNA damage from asbestos fibers is thought to be an important pathogenic pathway leading to mesothelioma. When mesothelial cells engulf asbestos fibers, the fibers can penetrate the nucleus and directly 
interfere with mitotic spindles and chromosomes. The interwoven complex of mitotic structures and asbestos fibers results in impaired mitosis, with structural chromosomal abnormalities and aneuploidy. ${ }^{31}$ As mentioned above, additional indirect DNA damage and DNA strand breakage may occur through asbestos-induced generation of ROS.

Asbestos-induced impairment of various arms of the immune system is thought to be an important factor in malignant transformation. Studies in mice have shown that asbestos exposure decreases the cytotoxic activity and the overall number of natural killer (NK) cells in the pulmonary interstitium. ${ }^{43}$ Decreased activity of NK cells and an increase in NK cell number was seen in the peripheral blood of retired asbestos cement workers without symptomatic lung disease, while decreased cytotoxic activity of NK cells in humans with asbestosis has been observed. ${ }^{44} 45$ Several human studies have shown increased serum levels of immunoglobulins in asbestos-exposed subjects. Mouse studies have shown that genetically immunodeficient mice show higher numbers of cells (predominantly neutrophils) in pulmonary lavage fluid along with increased severity of pulmonary lesions after asbestos inhalation compared with immunologically normal mice with the same exposure or genetically immunodeficient mice that have been reconstituted with lymphocytes. ${ }^{46}$ It is possible that genetically determined 'robustness' of the immune system is an important determinant in the observed variability for disease development after asbestos exposure.

The contribution of genetics to the development of mesothelioma is an area under active investigation. Germline mutations are rare in mesothelioma, being present in $1-5$ per cent of selected cases, ${ }^{47}$ but they suggest an inherited predisposition to developing mesothelioma. For example, germline BRCA1-associated protein-1 (BAP1) mutations have been observed in a study of two families with a relatively high incidence of mesothelioma. ${ }^{48}$ The tumor suppressor gene BAP1 is an enzyme involved in epigenetic control of chromatin remodeling, which leads to chromosomal loss when mutated. ${ }^{47}$ Similarly, a study of patients with quantified asbestos exposure and pleural mesothelioma found approximately 10 per cent of study subjects carried germline mutations in a DNA-repair pathway gene. The genes that were identified in this study were PALB2, BRCA 1, FANCI, ATM, SLX4, BRCA2, FANCC, FANCF, $P M S 1$, and XPC. The patients that carried these mutations had lower overall levels of asbestos exposure than patients that did not have the mutations, suggesting that their ability to effectively repair asbestos-induced DNA damage was impaired. ${ }^{49}$

Somatic mutations are more commonly seen in mesothelioma. Importantly, BAP1 is the gene most commonly mutated in mesothelioma, with loss of the gene being reported in $>50$ per cent of human cases. ${ }^{47}$ Recent evidence has shown that pleural mesothelioma cell lines with loss of BAP1 displayed enhanced sensitivity to inhibition of fibroblast growth factor receptor. This suggests a potential target in patients with mesotheliomas and demonstrated BAP1 mutations. ${ }^{50}$

NF2 and CDKN2A are other somatic mutations seen in mesothelioma. ${ }^{47} \mathrm{NF} 2$ mutations are present in approximately 50 per cent of mesothelioma cases. ${ }^{47}$ NF2 encodes the protein Merlin, which is involved in several cellular signaling pathways, such as mammalian target for rapamycin (mTOR), focal adhesion kinase, and Hippo. Both Hippo and mTOR pathways are involved in cell proliferation and growth. ${ }^{31}$ Merlin-negative mesothelioma cells have been shown in vitro to be more sensitive to the MTOR inhibitor, rapamycin, than Merlin-positive cells. The Hippo pathway has been shown to be an important regulator in organ size and tissue homeostasis. It is involved in regulating apoptosis, stemness, and cell proliferation in response to a variety of signals including cell-cell contact, cell polarity, mechanical signals, ligands of G-protein-coupled receptors, and cellular energy status. Dysregulation of the Hippo pathway plays an important role in carcinogenesis. ${ }^{51} \mathrm{CDKN} 2 \mathrm{~A}$ mutations are present in approximately $27-50$ per cent of mesothelioma cases. CDKN2A mutations result in alterations in p53 and retinoblastoma pathways. ${ }^{47} \mathrm{NF} 2$ and CDKN2A are often co-inactivated in mesothelioma. ${ }^{52}$ Knockout mice that have loss of both NF2 and CDKN2A have demonstrated accelerated onset and progression of asbestos-induced mesothelioma compared with mice that were wild-type or with loss of NF2 alone. This indicates cooperativity between NF2 and CDKN2A in driving more aggressive mesothelioma phenotypes. ${ }^{52}$

\section{SCREENING FOR ASBESTOS-INDUCED MALIGNANCY}

Currently, there are no guidelines on screening asbestos-exposed individuals for mesothelioma. The best prevention of asbestos-related disease is asbestos abatement programs, which have substantially reduced the incidence of asbestos-related diseases. The challenge is identifying patients that are at the highest risk of developing malignant disease and require close monitoring. While the majority of asbestos exposure occurs in the occupational setting, limiting screening to these individuals is insufficient. As highlighted by our case, non-occupational exposure, such as coming into close contact with the clothes of asbestos workers, is a recognized mechanism of exposure. Similarly, absolute dose is not a clear criterion for who should be regularly screened for malignancy. In general, there is a dose-response relationship between amount of asbestos exposure and risk of developing lung cancer and mesothelioma. However, this relationship is not the sole determinant of cancer risk. There are likely genetic factors that determine individual risk of development of asbestos-related benign disease, asbestos-related malignancy, or both. Our case highlights the probable genetic determinants in the development of asbestos-related lung disease and presents an interesting example of variable expression of asbestos-related disease within the same family. Our patient's father, with the largest asbestos exposure, had no disease, her mother died from mesothelioma, and the patient expressed a mixed phenotype of pleural plaques without cancer development. Setting a time limit for screening is also problematic. While lung carcinoma and mesothelioma are thought to result from long-term asbestos exposure, studies in both humans and mice have shown that these malignancies may occur after exposure periods as short as $1-12$ months. ${ }^{4}$

Controversy exists whether or not the presence of pleural plaques predisposes to developing mesothelioma, and subsequently, if patients identified with pleural plaques should be more closely monitored. Pleural plaques have 


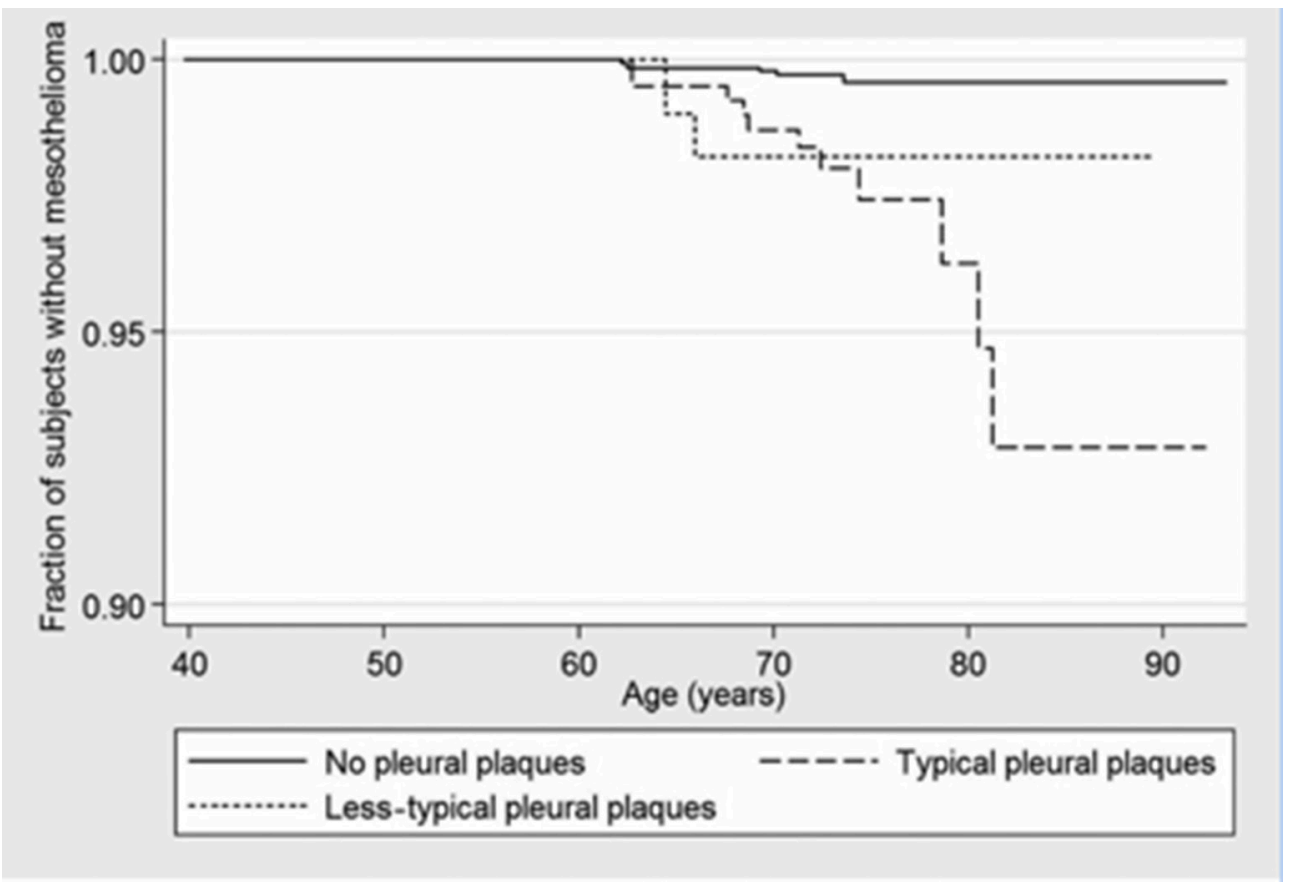

Figure 3 Proportion of asbestos-exposed individuals without mesothelioma at any given age stratified by presence or absence of pleural plaques (Pairon et al) $)^{53}$.

been seen in people with relatively low cumulative exposures to asbestos. ${ }^{4}$ Pleural plaques are traditionally considered benign entities, though there is suggestion that that their presence identifies asbestos-exposed individuals at higher risk for developing mesothelioma. A study that enrolled patients with former asbestos exposure to undergo screening for asbestos-related diseases found an association between pleural plaques and mesothelioma with a statistically significant HR of 8.9 (figure 3 ). ${ }^{53}$

Better screening methods will likely rely on advanced understanding of asbestos-induced malignant transformation. While imaging has traditionally been used to evaluate for mesothelioma, serum biomarkers are an emerging modality that may be useful in monitoring asbestos-exposed patients for mesothelioma. These biomarkers include mesothelin-related protein, osteopontin, and megakaryocyte potentiating factor. ${ }^{54}$ Serum osteopontin levels have been shown to distinguish asbestos-exposed individuals without mesothelioma from asbestos-exposed individuals with mesothelioma in one study. ${ }^{55}$ Other studies have shown osteopontin to have poor specificity for mesothelioma. ${ }^{56}$ In various studies, soluble mesothelin family proteins have shown good specificity for mesothelioma, but poor sensitivity. ${ }^{56}$ While these biomarkers hold promise, they have limitations. Further work is needed to validate these biomarkers, establish cut-points that optimize sensitivity and specificity, and define the role of combining different biomarkers as well as imaging to screen for mesothelioma. Further research objectives should include the development of screening tests and strategies that identify patients at higher risk of developing asbestos-related malignancy and early biomarkers of disease development.

Contributors Both authors conceptualized, wrote, and revised the article. ES is the guarantor of the article and performed literature search.
Competing interests None declared.

Provenance and peer review Not commissioned; externally peer reviewed.

(c) American Federation for Medical Research (unless otherwise stated in the text of the article) 2018. All rights reserved. No commercial use is permitted unless otherwise expressly granted.

\section{REFERENCES}

$1 \mathrm{NIH}$. Asbestos Exposure and Cancer Risk: National Cancer Institute, 2009. www.cancer.gov

2 Liu G, Cheresh P, Kamp DW. Molecular basis of asbestos-induced lung disease. Annu Rev Pathol 2013;8:161-87.

3 Rosenstock L, Hudson L. Nonmalignant asbestos-induced pleural disease. Semin Respir Crit Care Med 1986;7:197-202.

4 Toxicological Profile for Asbestos. Agency for Toxic Substances and Disease Registry, Sep 2001, 2016. www.atsdr.cdc.gov

5 Baker EL, Dagg T, Green RE. Respiratory illness in the construction trades. J Occup Med 1985;27:483-9.

6 Oliver LC, Eisen EA, Greene R, et al. Asbestos-related pleural plaques and lung function. Am J Ind Med 1988;14:649-56.

7 Hjortsberg U, Orbaek P, Aborelius M, et al. Railroad workers with pleural plaques: I. Spirometric and nitrogen washout investigation on smoking and nonsmoking asbestos-exposed workers. Am J Ind Med 1988;14:635-41.

8 Hedenstierna G, Alexandersson R, Kolmodin-Hedman B, et al. Pleural plaques and lung function in construction workers exposed to asbestos. Eur J Respir Dis 1981;62:111-22.

9 Steele M, Asbestosis SD, Fibrosis A-IP. In: Schwarz M, King T, Interstitial Lung Disease. 5th ed. USA: People's Medical Publishing House, 2011:543-55.

10 Taskinen E, Ahlamn K, Wükeri M. A current hypothesis of the lymphatic transport of inspired dust to the parietal pleura. Chest 1973;64:193-6.

11 Lee KP. Lung response to particulates with emphasis on asbestos and other fibrous dusts. Crit Rev Toxicol 1985;14:33-86.

12 Morgan A, Evans JC, Holmes A. Deposition and clearance of inhaled fibrous minerals in the rat studies using radioactive tracer techniques. Environ Res 1975; 10:196-207.

13 Sebastien P, Fondimare A, Bignon J, et al. Topographic distribution of asbestos fibres in human lung in relation to occupational and non-occupational exposure. Inhaled Part 1975;4 Pt 2:435-46. 
14 McLoud TC, Woods BO, Carrington CB, et al. Diffuse pleural thickening in an asbestos-exposed population: prevalence and causes. AJR Am J Roentgenol 1985:144:9-18.

15 Epler GR, McLoud TC, Gaensler EA. Prevalence and incidence of benign asbestos pleural effusion in a working population. JAMA 1982;247:617.

16 Murphy RL, Becklake MR, Brooks SM, et al. The diagnosis of nonmalignant diseases related to asbestos. Am Rev Respir Dis 1986:134:363-8.

17 Affairs COS. A physician's guide to asbestos-related diseases. JAMA 1984:252:2593-7.

18 Berry G. Mortality of workers certified by pneumoconiosis medical panels as having asbestosis. Br J Ind Med 1981;38:130-7.

19 Mattson SB. Monosymptomatic exudative pleurisy in persons exposed to asbestos dust. Scand J Respir Dis 1975;56:263.

20 Kato K, Gemba K, Fujimoto N, et al. Pleural irregularities and mediastinal pleural involvement in early stages of malignant pleural mesothelioma and benign asbestos pleural effusion. Eur J Radiol 2016;85:1594-600.

21 Brody AR, Hill LH, Adkins B, et al. Chrysotile asbestos inhalation in rats: deposition pattern and reaction of alveolar epithelium and pulmonary macrophages. Am Rev Respir Dis 1981;123:670-9.

22 Quinlan TR, BéruBé KA, Marsh JP, et al. Patterns of inflammation, cell proliferation, and related gene expression in lung after inhalation of chrysotile asbestos. Am J Pathol 1995;147:728-39.

23 Wright JL, Cagle P, Churg A, et al. Diseases of the small airways. Am Rev Respir Dis 1992;146:240-62.

24 Burkhardt A. Alveolitis and collapse in the pathogenesis of pulmonary fibrosis. Am Rev Respir Dis 1989;140:513-24.

25 Haugen A, Schafer PW, Lechner JF, et al. Cellular ingestion, toxic effects, and lesions observed in human bronchial epithelial tissue and cells cultured with asbestos and glass fibers. Int J Cancer 1982;30:265-72.

26 Chang LY, Overby LH, Brody AR, et al. Progressive lung cell reactions and extracellular matrix production after a brief exposure to asbestos. Am J Pathol 1988;131:156-70.

27 Warheit DB, Hill LH, George G, et al. Time course of chemotactic factor generation and the corresponding macrophage response to asbestos inhalation. Am Rev Respir Dis 1986;134:128-33.

28 Cole RW, Ault JG, Hayden JH, et al. Crocidolite asbestos fibers undergo sizedependent microtubule-mediated transport after endocytosis in vertebrate lung epithelial cells. Cancer Res 1991;51:4942-7.

29 Kamp DW, Graceffa P, Pryor WA, et al. The role of free radicals in asbestosinduced diseases. Free Radic Biol Med 1992:12:293-315.

30 Lund LG, Aust AE. Iron-catalyzed reactions may be responsible for the biochemical and biological effects of asbestos. Biofactors 1991;3:83-9.

31 Sekido Y. Molecular pathogenesis of malignant mesothelioma. Carcinogenesis 2013:34:1413-9.

32 Thomas G, Ando T, Verma K, et al. Asbestos fibers and interferon-gamma upregulate nitric oxide production in rat alveolar macrophages. Am J Respir Cell Mol Biol 1994;11:707-15.

33 Chao CC, Park SH, Aust AE. Participation of nitric oxide and iron in the oxidation of DNA in asbestos-treated human lung epithelial cells. Arch Biochem Biophys 1996;326:152-7.

34 Choe N, Tanaka S, Kagan E. Asbestos fibers and interleukin-1 upregulate the formation of reactive nitrogen species in rat pleural mesothelial cells. Am J Respir Cell Mol Biol 1998;19:226-36.

35 Perderiset M, Marsh JP, Mossman BT. Activation of protein kinase C by crocidolite asbestos in hamster tracheal epithelial cells. Carcinogenesis 1991;12:1499-502
36 Janssen YM, Heintz NH, Mossman BT. Induction of c-fos and c-jun protooncogene expression by asbestos is ameliorated by N-acetyl-L-cysteine in mesothelial cells. Cancer Res 1995;55:2085-9.

37 Mullin JM, O'Brien TG. Effects of tumor promoters on LLC-PK1 renal epithelial tight junctions and transepithelial fluxes. Am J Physiol 1986;251:C597-C602.

38 Winter M, Wilson JS, Bedell K, et al. The conductance of cultured epithelial cell monolayers: oxidants, adenosine triphosphate, and phorbol dibutyrate. Am J Respir Cell Mol Biol 1990;2:355-63.

39 Holian A, Kelley K, Hamilton RF. Mechanisms associated with human alveolar macrophage stimulation by particulates. Environ Health Perspect 1994;102:69-74.

40 Heintz NH, Janssen YM, Mossman BT. Persistent induction of c-fos and c-jun expression by asbestos. Proc Natl Acad Sci U S A 1993;90:3299-303.

41 Angel P, Karin M. The role of Jun, Fos and the AP-1 complex in cell-proliferation and transformation. Biochim Biophys Acta 1991;1072:129-57.

42 Janssen YM, Barchowsky A, Treadwell M, et al. Asbestos induces nuclear factor kappa B (NF-kappa B) DNA-binding activity and NF-kappa B-dependent gene expression in tracheal epithelial cells. Proc Natl Acad Sci U SA 1995:92:8458-62.

43 Rosenthal GJ, Corsini E, Simeonova P. Selected new developments in asbestos immunotoxicity. Environ Health Perspect 1998;106(Suppl 1):159-69.

44 Froom P, Lahat N, Kristal-Boneh E, et al. Circulating natural killer cells in retired asbestos cement workers. J Occup Environ Med 2000;42:19-24.

45 Kubota M, Kagamimori S, Yokoyama K, et al. Reduced killer cell activity of lymphocytes from patients with asbestosis. Br J Ind Med 1985;42:276-80.

46 Corsini E, Luster MI, Mahler J, et al. A protective role for T lymphocytes in asbestos-induced pulmonary inflammation and collagen deposition. Am J Respir Cell Mol Biol 1994;11:531-9.

47 Napolitano A, Carbone M. Malignant Mesothelioma: Time to Translate? Trends Cancer 2016;2:467-74.

48 Cheung M, Testa JR. BAP1, a tumor suppressor gene driving malignant mesothelioma. Trans/ Lung Cancer Res 2017;6:270-8.

49 Betti M, Casalone E, Ferrante D, et al. Germline mutations in DNA repair genes predispose asbestos-exposed patients to malignant pleural mesothelioma. Cancer Lett 2017;405:38-45.

50 Quispel-Janssen JM, Badhai J, Schunselaar L, et al. Comprehensive pharmacogenomic profiling of malignant pleural mesothelioma identifies a subgroup sensitive to FGFR inhibition. Clin Cancer Res 2017. doi:10.1158/1078-0432.CCR-17-1172. [Epub ahead of print 23 Oct 2017].

51 Yu FX, Zhao B, Guan KL. Hippo Pathway in Organ Size Control, Tissue Homeostasis, and Cancer. Cell 2015;163:811-28.

52 Menges CW, Kadariya Y, Altomare D, et al. Tumor suppressor alterations cooperate to drive aggressive mesotheliomas with enriched cancer stem cells via a p53-miR-34a-c-Met axis. Cancer Res 2014;74:1261-71.

53 Pairon JC, Laurent F, Rinaldo M, et al. Pleural Plaques and the risk of Pleural mesothelioma. J Nat/ Cancer Inst 2013;105:293-301.

54 Pass $\mathrm{HI}$, Carbone M. Current status of screening for malignant pleural mesothelioma. Semin Thorac Cardiovasc Surg 2009;21:97-104.

55 Pass HI, Lott D, Lonardo F, et al. Asbestos exposure, pleural mesothelioma, and serum osteopontin levels. N Engl J Med 2005;353:1564-73.

56 Pantazopoulos I, Boura P, Xanthos T, et al. Effectiveness of mesothelin family proteins and osteopontin for malignant mesothelioma. Eur Respir J 2013:41:706-15. 